\title{
Spatial and Functional Relationships Between Air Conduits and Blood Capillaries in the Pulmonary Gas Exchange Tissue of Adult and Developing Chickens
}

\author{
ANDREW N. MAKANYA, ${ }^{1,2}$ YOSIF EL-DARAWISH, ${ }^{1}$ BONIFACE M. KAVOI, ${ }^{2}$ AND VALENTIN DJONOV ${ }^{1 *}$ \\ ${ }^{1}$ Institute of Anatomy, Department of Medicine, University of Fribourg, Rte Albert Gockel 1, CH-1700, Fribourg, Switzerland \\ ${ }^{2}$ Department of Veterinary Anatomy and Physiology, University of Nairobi, Riverside Drive, P. O. Box 30197-00100, Nairobi, Kenya
}

\begin{abstract}
KEY WORDS chick; avian lung; air capillaries; blood capillaries; three-dimensional; crosscurrent
\end{abstract}

\begin{abstract}
The documented data regarding the three-dimensional structure of the air capillaries $(\mathrm{ACs})$, the ultimate sites of gas exchange in the avian lung is contradictory. Further, the mode of gas exchange, described as cross-current has not been clearly elucidated. We studied the temporal and spatial arrangement of the terminal air conduits of the chicken lung and their relationship with the blood capillaries (BCs) in embryos as well as the definitive architecture in adults. Several visualization techniques that included corrosion casting, light microscopy as well as scanning and transmission electron microscopy were used. Two to six infundibulae extend from each atrium and give rise to numerous ACs that spread centrifugally. Majority of the ACs are tubular structures that give off branches, which anastomose with their neighboring cognates. Some ACs have globular shapes and a few are blind-ending tapering tubes. During inauguration, the luminal aspects of the ACs are characterized by numerous microvillus-like microplicae, which are formed during the complex processes of cell attenuation and canalization of the ACs. The parabronchial exchange BCs, initially inaugurated as disorganized meshworks, are reoriented via pillar formation to lie predominantly orthogonal to the long axes of the ACs. The remodeling of the retiform meshworks by intussusceptive angiogenesis essentially accomplishes a cross-current system at the gas exchange interface in the adults, where BCs form ring-like patterns around the ACs, thus establishing a crosscurrent system. Our findings clarify the mode of gas exchange in the parabronchial mantle and illuminate the basis for the functional efficiency of the avian lung.
\end{abstract}

\section{INTRODUCTION}

The avian lung is a structurally complex, albeit functionally efficient organ that has baffled researchers over the years. Although the precise arrangement of the large air conduits in the lung of the domestic fowl is well documented (Makanya and Djonov, 2008), the literature on the $3 \mathrm{D}$ architecture of the blood capillaries $(\mathrm{BCs})$ and air capillaries (ACs) is contradictory. Though the documented information on the functional design of the avian respiratory system has remained confused for a long time, recent efforts to elucidate its architecture appear to be bearing fruit (Maina, 2003; Makanya and Djonov, 2008; Woodward and Maina, 2005,2008 ). For such a complex structure as the avian lung, use of several visualization techniques has overt advantages (Makanya and Djonov, 2008) while investigations during embryogenesis allow a clearer understanding of the incipient structures (Locy and Larsell, 1916; Makanya and Djonov, 2008, 2009).

In a recent publication we have indicated that in the mature chicken lung, the general orientation of the $\mathrm{BCs}$ with respect to ACs at the gas exchange interface is orthogonal (Makanya and Djonov, 2009). However, details of how the ACs radiate in space and how they interact with the BCs were not investigated. In concerted efforts, Woodward and Maina $(2005,2008)$ have used 3D reconstruction in an effort to illustrate the spatial relationship between ACs and the BCs in the gas exchange tissue of the mallard duck. Several previous researchers have investigated the structure of the parabronchial unit of the avian lung (Duncker, 1978; Klika et al., 1997, 1998; Scheuermann et al., 1997), but the precise structure and arrangement of the gas exchanging units remains elusive. In a comprehensive review, Duncker (1978) reported on the morphological aspects of the amniotic lung with the notion that the smooth muscles in the luminal aspects of the interatrial septa function to regulate flow of air through the parabronchi. The outer margins of atria are lined by the anastomosing interatrial septa and the atrial floor has openings for 3-6 infundibulae (Scheuermann et al., 1998). Although the cells lining the atria, infundibulae, and the ACs are well studied, less is known

*Correspondence to: Valentin Djonov, Institute of Anatomy, Department of Medicine, University of Fribourg, Rte Albert Gockel 1, CH-1700, Fribourg, Switzerland. E-mail: valentin.djonov@unifr.ch

Received 10 February 2010; accepted in revised form 2 May 2010

Contract grant sponsor: Swiss National Science Foundation; Contract grant number: 31-003A-116243 
regarding spatial distribution of the air conduits and BCs in the exchange tissue. Several studies have insinuated that at the air-capillary level, blood flow may be counter-current [see reviews by Maina (2006, 2008) and references therein] while others suggest that it is cross-current (Abdalla and King, 1975; Duncker, 1971; Nasu, 2005; West et al., 1977). Nevertheless, empirical evidence of such an arrangement has been only briefly presented and only in a few studies (Makanya and Djonov, 2009; Nasu, 2005; West et al., 1977). An unequivocal demonstration of the spatial relationship between these two categories of conductive structures has not been presented, the closest being $3 \mathrm{D}$ reconstruction that indicates that portions of the $\mathrm{ACs}$ are globular, some are interconnected while others are solitary (Woodward and Maina, 2005). The latter authors also report that the BCs form a meshwork of short segments, but as the authors note themselves, the techniques have their inherent limitations (Woodward and Maina, 2005).

The nomenclature and topographical arrangement of the parabronchial vasculature has recently been updated, with the conclusion that while arterial blood generally flows centripetally toward the parabronchial lumen, the bulk of the exchange capillaries are orthogonal to the ACs (Makanya and Djonov, 2009). In the current study, we have used microscopic and casting techniques to study the spatial relationship between blood and ACs in the parabronchial gas exchange mantle in embryonic and adult birds. Our results indicate that at the exchange level, the arrangement is predominantly cross-current.

\section{MATERIALS AND METHODS Animal Provenance}

Adult Rhode Island Red layers (Gallus gallus variant domesticus) already identified for culling were purchased from farmers. Chick embryos were grown from Brown Leghorn eggs obtained from commercial breeders. Eggs were incubated at $37^{\circ} \mathrm{C}$ and at a humidity of $65 \%$. Embryos covering Hamburger and Hamilton $(\mathrm{HH})$ stages 43-46 (embryonic days 16-21) were obtained and processed as detailed below. A summary of the techniques and the numbers of specimens used for each technique is provided in Table 1 . In all cases, anesthesia was achieved by intra-abdominal injection of sodium pentobarbitone. All protocols were approved by the Animal Care and Use Committee of the University of Nairobi.

\section{Light and Electron Microscopy}

For light microscopy (LM), transmission (TEM), and scanning (SEM) electron microscopy investigations, lungs were fixed in situ by intratracheal infusion of a solution of $2.5 \%$ glutaraldehyde in $0.1 \mathrm{M}$ cacodylate buffer $\left(\mathrm{pH} \mathrm{7.4,} 350 \mathrm{mOsmol} / \mathrm{kgH}_{2} \mathrm{O}\right)$. Lungs were dissected out and diced into small blocks. Tissue blocks were postfixed in osmium tetroxide, block-stained using uranyl acetate, dehydrated through ascending concentrations of ethanol, and embedded in epoxy resin. Semithin sections were obtained at a nominal thickness of $1 \mu \mathrm{m}$, stained with toluidine blue, and viewed under a Coolscope Digital Microscope (Nikon) or Leica DMBR digital light microscope. Ultrathin sections were obtained at $90 \mathrm{~nm}$, counterstained with lead
TABLE 1. Summary of the techniques used to visualize the $3 D$ arrangement of ACs and BCs in the lung of the domestic fowl

\begin{tabular}{clcc}
\hline $\begin{array}{l}\text { Technique } \\
\text { no. }\end{array}$ & \multicolumn{1}{c}{ Technique description } & $\begin{array}{c}\text { Age }(\mathrm{s}) \\
\text { studied }\end{array}$ & $\begin{array}{c}\text { Number of } \\
\text { specimens }\end{array}$ \\
\hline 1 & $\begin{array}{l}\text { Critical-point dried } \\
\text { tissues and SEM }\end{array}$ & E20 & 4 \\
& Semithin sections & E21 & 5 \\
2 & Paraffin sections with $\alpha$-SMA & E21 & 3 \\
3 & Intratracheal casting and SEM & E16 & 3 \\
4 & & E19 & 3 \\
& & Ad & 4 \\
5 & TEM & E16 & 5 \\
& & E18 & 5 \\
& & E19 & 5 \\
6 & Intravascular casting and SEM & E20 & 3 \\
& & Ad & 3 \\
7 & Combined intravascular and & Ad & 3 \\
& intratracheal casting with SEM & & \\
\hline
\end{tabular}

The number of specimens used for each technique is also provided. The letter "E" denotes embryonic age in days while "Ad" refers to adults.

citrate, and viewed on a Philips EM-300 microscope. For the SEM, the lungs were fixed by intratracheal infusion of the same fixative as above. Selected lung specimens were dehydrated through ascending concentrations of ethanol, critical point-dried in liquid carbon dioxide, and mounted on aluminum stubs. The specimens were sputter-coated with gold and viewed under a Philips XL 30 FEG scanning electron microscope or a Philips XL 30 SFEG scanning electron microscope.

\section{Immunohistochemistry}

Lungs from E21 embryos were fixed by intratracheal infusion of $4 \%$ paraformaldehyde. The lungs were then dissected out and immersed in the same fixative for 2 $\mathrm{h}$, rinsed in $15 \%$ sucrose solution, and then stored in $70 \%$ ethanol. Subsequently, the lungs were processed as detailed previously (Makanya et al., 2006). Briefly, tissue blocks were embedded in paraffin wax and cut at a nominal thickness of $3 \mu \mathrm{m}$. Paraffin sections were transferred to gelatinized microslides and air-dried overnight at $37^{\circ} \mathrm{C}$. The sections were dewaxed in xylene (three changes), rehydrated in graded series of ethanol, and rinsed twice in Tris-buffered saline (TBS) $50 \mathrm{mM}$ Tris/HCl (pH 7.4) containing $100 \mathrm{mM}$ sodium chloride. After incubation with the first antibody diluted in TBS (mouse anti $\alpha$-SMA 1:200; Sigma, Buchs, Switzerland), sections were exposed to an affinity-purified biotinylated second antibody (antimouse EO 433, Dako; Glostrup, Denmark; diluted 1:200 in TBS) for $45 \mathrm{~min}$ at ambient temperature, washed three times in TBS, then treated with the avidin-biotin-horseradish peroxidase complex (P355, Dako) for a similar period. The sections were exposed to 3-amino-9-ethylcarbazole or 3,3-diaminobenzidine (Sigma Chemicals Company, St. Louis, MO) for 10 min and viewed in a Coolscope Digital Microscope (Nikon). Negative controls were prepared using nonspecific mouse sera.

\section{Intravascular Casting}

Lungs from three adult birds and E16-E21 (HH stages 43-46) embryos (at least three per time point) were used for microscopic observations of the parabronchial vessels. Embryos were cultured shell-free in petri-dishes and appropriate $\mathrm{HH}$ stages selected for vascular casting. The embryos were perfused with a so- 
lution of $0.9 \%$ sodium chloride containing $1 \%$ Liqemin and $1 \%$ procaine through the vitelline artery. The vasculature was then filled with methylmethacrylate resin (Mercox, Vilene Hospital, Japan) containing $0.1 \mathrm{~mL}$ accelerator per every $5 \mathrm{~mL}$ of the resin or with Technovit $^{\circledR} 3040$ powder mixed with catalyst in the ratio 1:1 as per the manufacturer's instructions. One hour after perfusion, lungs were dissected out and immersed in Ringer's solution for at least $2 \mathrm{~h}$ and subsequently transferred to a $15 \%$ potassium hydroxide solution for 2-4 weeks. After dissolution of the tissues, the embryos and organs were washed, dehydrated in ascending concentrations of ethanol, and dried in a desiccator. Samples were mounted on aluminum stubs, sputter-coated with gold, and viewed under a Philips XL 30 FEG or a Philips XL 30 SFEG scanning electron microscope. For the adult birds, perfusion was either done through the pulmonary artery or through the right ventricle of the heart then the lungs were filled with methyl methacrylate resin, dissected out and processed for SEM as described above.

\section{Intratracheal Casting}

Methylmethacrylate resin was introduced through the trachea of selected embryos and adults until the whole airway system was filled with the resin. At least $1 \mathrm{~h}$ after perfusion, the lungs were dissected out and then processed further for the scanning electron microscope as detailed above.

\section{Double Casting}

A combined intravascular and intratracheal casting (starting first with the vasculature) was performed on three adult birds to visualize the spatial relationships between the BCs and the ACs. Methylmethacrylate resin was introduced first through the left ventricle and subsequently through the tracheae. The entire pulmonary vasculature was filled with methylmethacrylate resin and allowed to set for at least $1 \mathrm{~h}$. The lungs were then dissected out and processed for the scanning electron microscope as detailed above.

\section{RESULTS}

An overview of the air conduits in the critical pointdried lung tissue at E20 chick embryo lung is presented in Figure 1. A bird's-eye view of the air conduits from the luminal aspect of the parabronchus at this stage (Figs. 1A and 1B) indicates that all the gas exchange conduits are well formed with atria giving rise to 3-6 infundibulae, which then form numerous ACs that radiate toward the periphery of the parabronchial wall. Longitudinal sections through the parabronchial mantle indicate that the infundibulae extend for a rather short distance and branch to give rise to several ACs. ACs start to branch as soon as they emerge from the infundibulae (Figs. 1C and 1D). A transection parallel to the long axes of the ACs obtained at the periphery of the parabronchial mantle (Figs. $1 \mathrm{E}$ and $1 \mathrm{~F}$ ) shows that ACs tend to be narrower toward the periphery where several anastomoses are also formed.

Investigations of the disposition of the air conduits at light microscopy (Fig. 2) show that the atria are not associated with exchange $\mathrm{BCs}$, but are shallow receptacles that direct air into and out of the infundibulae. The boundaries between infundibulae and ACs have no landmarks and the two structures continue into each other inconspicuously. The interatrial septa separate adjacent atria and are devoid of exchange capillaries (Figs. 2A and 2B). The sectioned ACs and infundibulae generate profiles of different shapes in $2 \mathrm{D}$, indicating the diversity in their geometry and at this level, AC branching was evident (Figs. 2C and 2D). The atria are supported by bundles of smooth muscle cells at the "tips" of the interatrial septa (Figs. 2E and 2F). These smooth muscle cells stained positively for $\alpha$-SMA, a strong indicator of their contractile ability. Notably such cells were restricted to the apical parts of the interatrial septa, and the walls of large blood vessels but were absent from the interparabronchial septa.

To clarify the spatial distribution of the air conduits, intratracheal mercox casting was done in developing (Figs. 3A-3D) and adult chickens (Figs. 3E and 3F). The infundibulae are well formed by E16 and many of them show the primordial AC sprouts (Fig. 3A) and by E19 these new ACs have formed secondary branches, some of which start to anastomose with neighboring cognates (Figs. 3C and 3D). Casts of the ACs at this latter stage have tiny holes, which represent the spaces occupied by intraluminal microplicae (Fig. 3D, see also Fig. 4). In the adult chicken lung, some ACs are slender branching tubes that taper toward the periphery of the parabronchus while others have globular shapes (Fig. $3 \mathrm{E}$ ). A double mercox cast (Fig. $3 \mathrm{~F}$ ) illustrates the intimate adherence of the BCs to the ACs in which an orthogonal relationship is accomplished.

The processes leading to spread and canalization of the ACs during development were captured at TEM level. Longitudinal sections of the ACs show several aposomes and numerous microplicae lining the inner aspect of the epithelium (Figs. 4A and 4B). In addition the apical parts of cells were associated with numerous vesicles, the rapture of which resulted in formation of the microplicae. In later stages of development (Figs. 4C and 4D), such microplicae and vesicles were seen to reduce in number as the thin blood-gas barrier was established by apposition of BCs to the incipient ACs.

The spatial relationships between the ACs on one hand and the BCs on the other, was studied by intravascular casting in embryos and adults. At E20 the capillary meshwork formed a reticulate pattern around the ACs (Figs. 5A and 5B), but when such were transected they showed a tendency to form almost circular rings around the $\mathrm{ACs}$, an indication of the formative cross-current system (Figs. 5C and 5D). A bird's-eye view of the developing vasculature from the parabronchial lumen reveals retiform baskets of irregularly shaped capillaries surrounding the ACs (Figs. 6A and $6 \mathrm{~B})$. At a closer view, such capillaries had numerous pillar holes the hallmark of intussusceptive angiogenesis (Fig. 6B). Such pillars were responsible for remodeling of the capillaries to ultimately assume a general orthogonal orientation with respect to the long axes of the ACs (Figs. 6C and 6D). The virtual absence of pillar holes and the cylindrical smooth nature of the BC casts in the adult lung underpin the crucial role of intussusceptive angiogenesis in the final angioarchitecture at the gas exchange region. An overview of the parabronchial vasculature from the external surface in the adult reveals large interparabronchial arteries running in the interparabronchial septum and giving rise to para- 

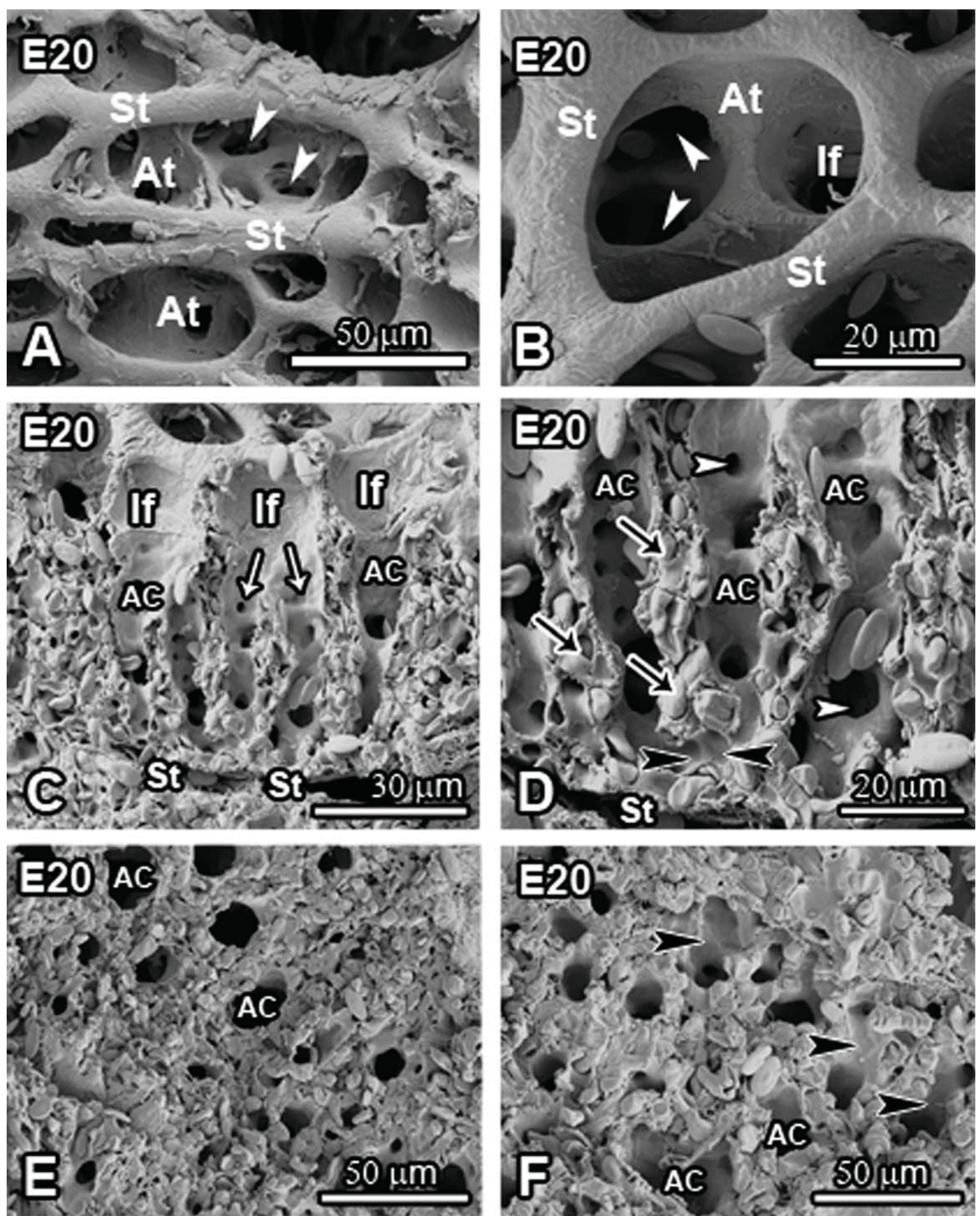

Fig. 1. SEM micrographs of E20 critical-point dried lung tissue illustrating the disposition of the air conduits in the parabronchial gas exchange area. A and $\mathbf{B}$ : A bird's-eye view of the atria (At) with the intervening interatrial septa (St). Atria open into infundibulae (If), which in turn give rise to air capillaries (open arrowheads). C and D: Sections through the parabronchial mantle showing the distribution of the air conduits. The infundibulae (If) project from the atria and extend for a short distance toward the periphery of the parabronchial mantle, then give rise to air capillaries (open arrows in C). The individual air capillaries (AC) form smaller branches (open arrowheads in D) that radiate into the parabronchial tissue. Toward the interparabronchial septum (St), the ACs anastomose (closed arrowheads in D). Notice the erythrocytes (closed arrows in D) lodged in BCs that occur in the septa separating adjacent ACs (see also E and F below). E and F: Profiles of the peripheral parts of the parabronchial mantle showing transected air capillaries (AC). Notice the anastomoses (closed arrowheads in F) connecting adjacent ACs.

bronchial arteries and some cases directly to parabronchial arterioles (Fig. 6E). At the peripheral aspects of the parabronchial mantle, an outer layer of BCs forms a close-knit capillary meshwork with circular rings around the tips of ACs (Fig. $6 \mathrm{~F}$ ), thus maintaining a cross-current system. 

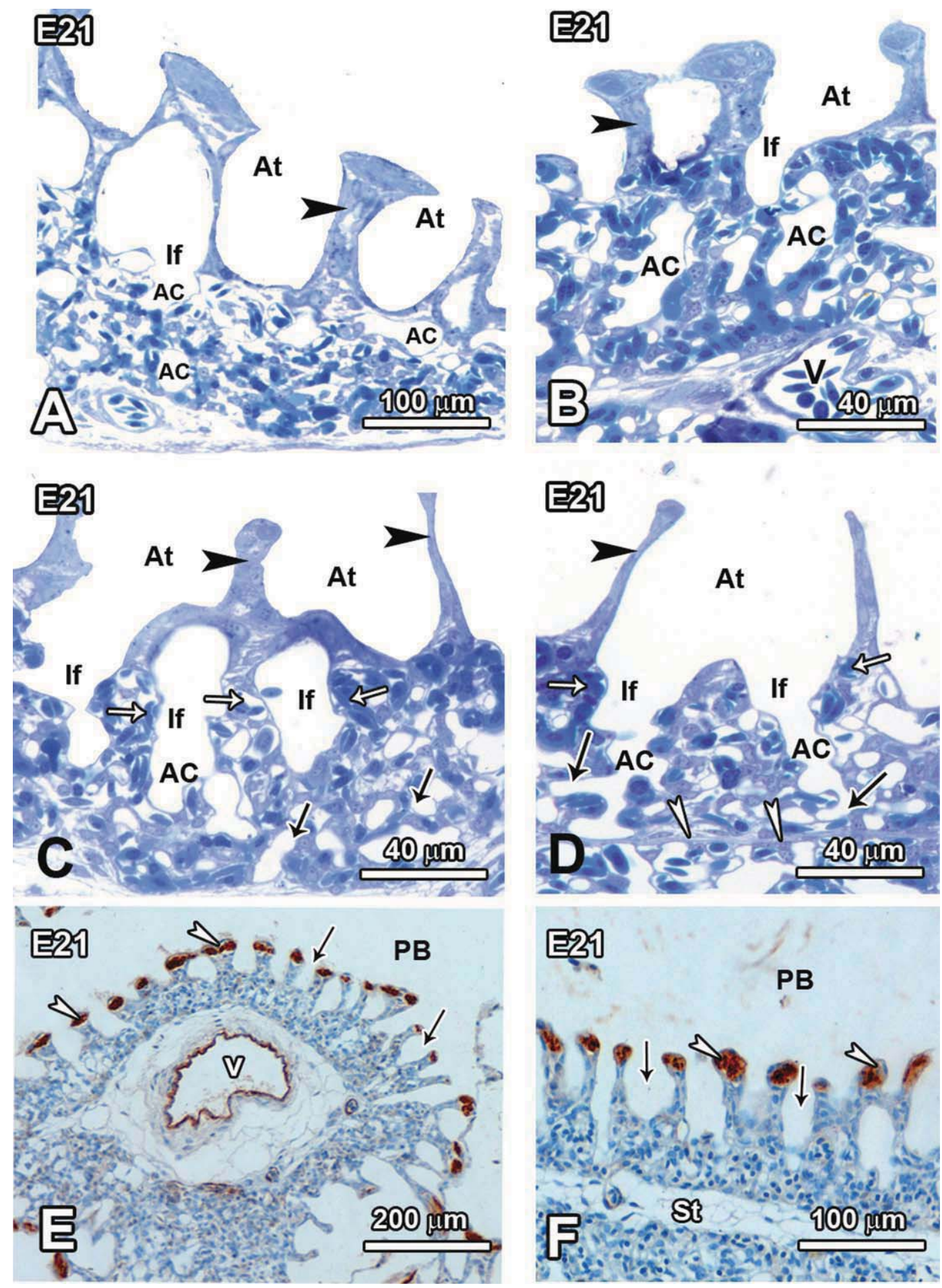

Fig. 2. Semithin (A-D) and paraffin (E and $\mathbf{F})$ sections of the E21 chick embryo showing the structure of the parabronchial air conduits. AD: The atria (At) are well delineated by interatrial septa (closed arrowheads). The atria give rise to short infundibulae (If), which then form air capillaries (AC) that taper toward the periphery of the parabronchus and give rise to smaller branches (closed arrows in C and D). Note that the ACs do not traverse the interparabronchial septum (open arrowheads in D), which is a narrow layer of connective tissue expanded only where there are large blood vessels (see V in B). The open arrows in $\mathrm{C}$ and $\mathrm{D}$ indicate that the infundibulae are associated with exchange capillaries. E and F: Paraffin sections through the parabronchial mantle showing the distribution of $\alpha$-smooth muscle actin ( $\alpha$-SMA). Apart from the walls of large blood vessels (V), $\alpha$-SMA positive cells are concentrated at the tips of atrial septa (open arrowheads) and are absent from the interparabronchial septa (St). Such cells surround the openings into the atria (closed arrows) and are probably important in regulation of airflow into and out of the atria. 

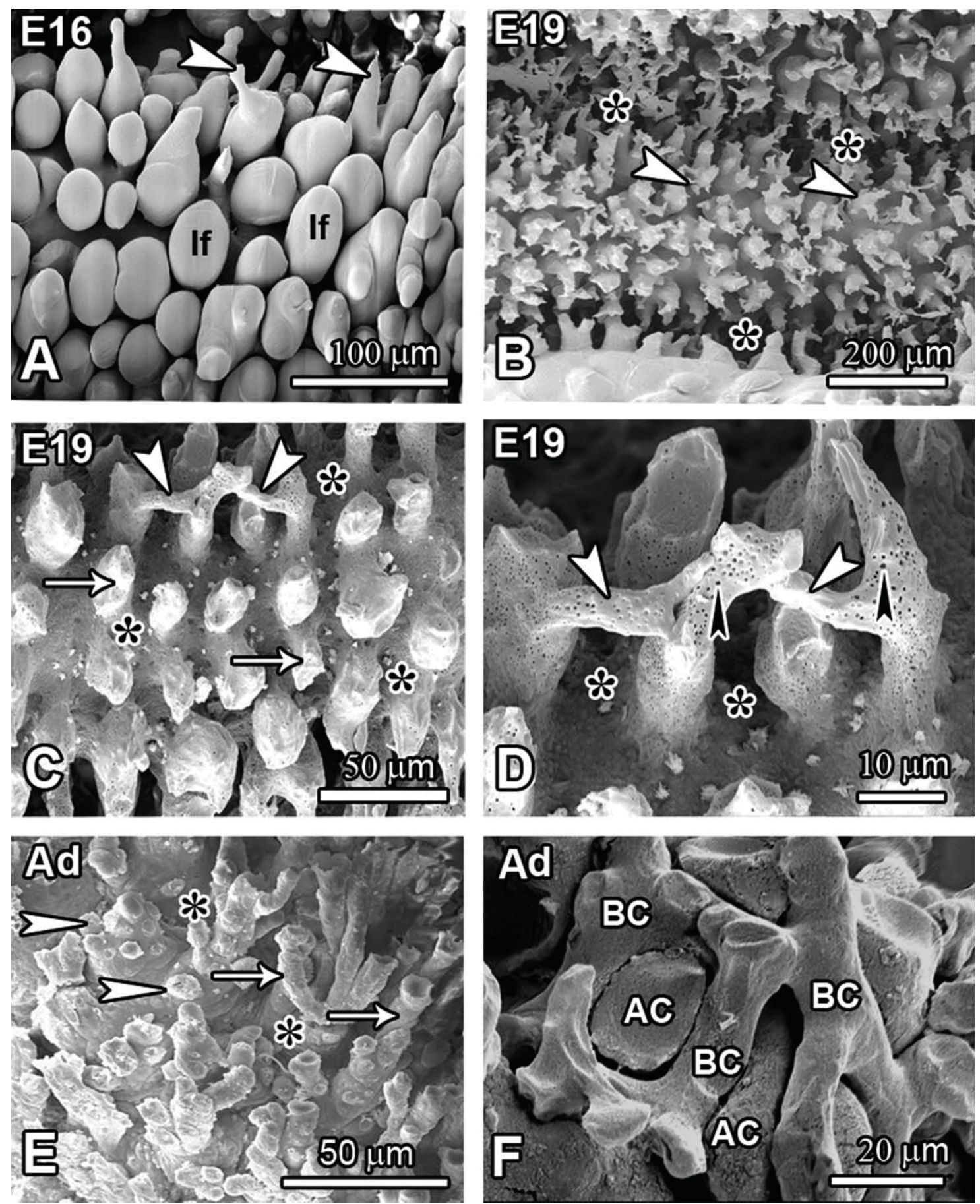

Fig. 3. Scanning electron micrographs of intratracheal mercox casts of the lungs of late stage embryos (E16-E19; A-D) and adult birds (E, F) showing the steps in formation of air capillaries and their branches as well as their definitive structures. A: Early at E16 the infundibulae (If) appear as smooth mounds on the parabronchial casts with only a few sprouting air capillaries (arrowheads). B-D: The ACs by E19 have started to form branches and some of such lateral branches extend to anastomose with neighboring ACs within the same parabronchus (open arrowheads in $\mathrm{C}$ and D). Asterisks in $\mathrm{B}$ represent the interparabronchial septa while those ones in $\mathrm{C}$ and D represent the space occupied by the exchange blood capillaries. The open arrows in C indicate unbranched ACs, while the closed arrowheads in D indicate tiny holes left in the ACs by microplicae (see also Fig. 4). E and F: In the adult (Ad) chicken, ACs are largely discrete branching structures that taper toward the periphery (open arrows in E) while some form globular shapes (open arrowheads in E). The asterisks in E denote spaces occupied by blood capillaries. A double mercox cast of the airway and vascular conduits shows that the blood capillaries (BC in F) tend to form rings around the air capillaries (AC). This essentially forms a cross-current system between the ACs and the BCs. 

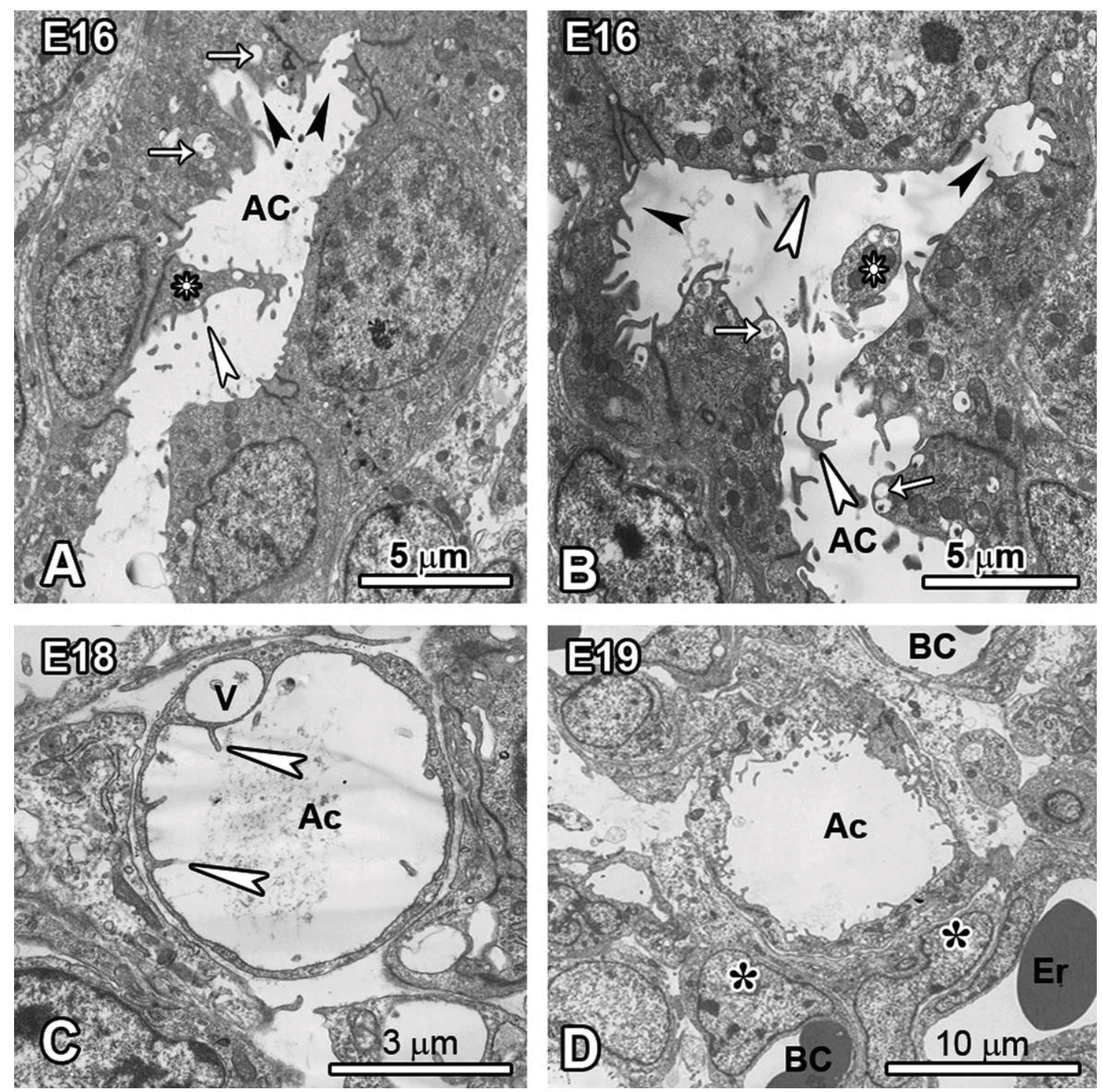

Fig. 4. TEM micrographs showing the developing air capillaries at E16-E19. A and B: At E16, the air capillaries contain both microplicae (open arrowheads) and intaluminal projections (stars) some of which appear to be floating bodies (star in B) in the lumen. Such structures result from cell attenuation processes collectively named secarecytosis, which in part proceed through vesicle and vacuole formation (arrows in $\mathrm{A}$ and $\mathrm{B}$ ) and their subsequent rapture resulting in formation of microplicae (open arrowheads). Furthermore, these attenuation mechanisms appear to be important in formation and canalization of the air capillary branches (see closed arrowheads). C and D: Cross sections of air capillaries at E18 (C) and E19 (D). Attenuation of the epithelium proceeds through many mechanisms that include vacuolation and vesiculation. Notice a vacuole (V) in the wall of the air capillary and microplicae (open arrowheads) that are the results of vacuolation and vesiculation. At this stage the ACs are approximated by blood capillaries (BC) to form a blood-gas barrier, which at this stage is still thick (asterisks in D). During these late stages of development, BCs are distinguished from ACs due to presence of erythrocytes (Er in D) and absence of microplicae.

\section{DISCUSSION}

In the current study, we have shown that ACs occur both in tubular tapering structures as well as globular or rounded shapes. The ACs form branches that radiate toward the periphery of the parabronchus and a majority of the branches anastomose with their neighboring cognates while a few are blind ending. Further, we have shown how the parabronchial BCs, initially inaugurated as disorganized meshworks (Makanya et al., 2007), are reoriented via pillar formation to lie predominantly orthogonal to the long axes of the ACs. This essentially establishes a cross-current system at the gas exchange level that augments the multicapillary serial arterialization, an arrangement that allows new deoxygenated blood to interact with air in successive regions of the parabronchus (Maina, 2006). The result is that a high concentration gradient of gases is maintained and hence efficient gas exchange. The multicapillary serial arterialization arrangement has recently been illustrated (Makanya and Djonov, 2009), and involves a design where successive regions of the parabronchus receive new deoxygenated blood from parabronchial arteries and are drained by intraparabronchial veins. Although the parabronchial arteries 

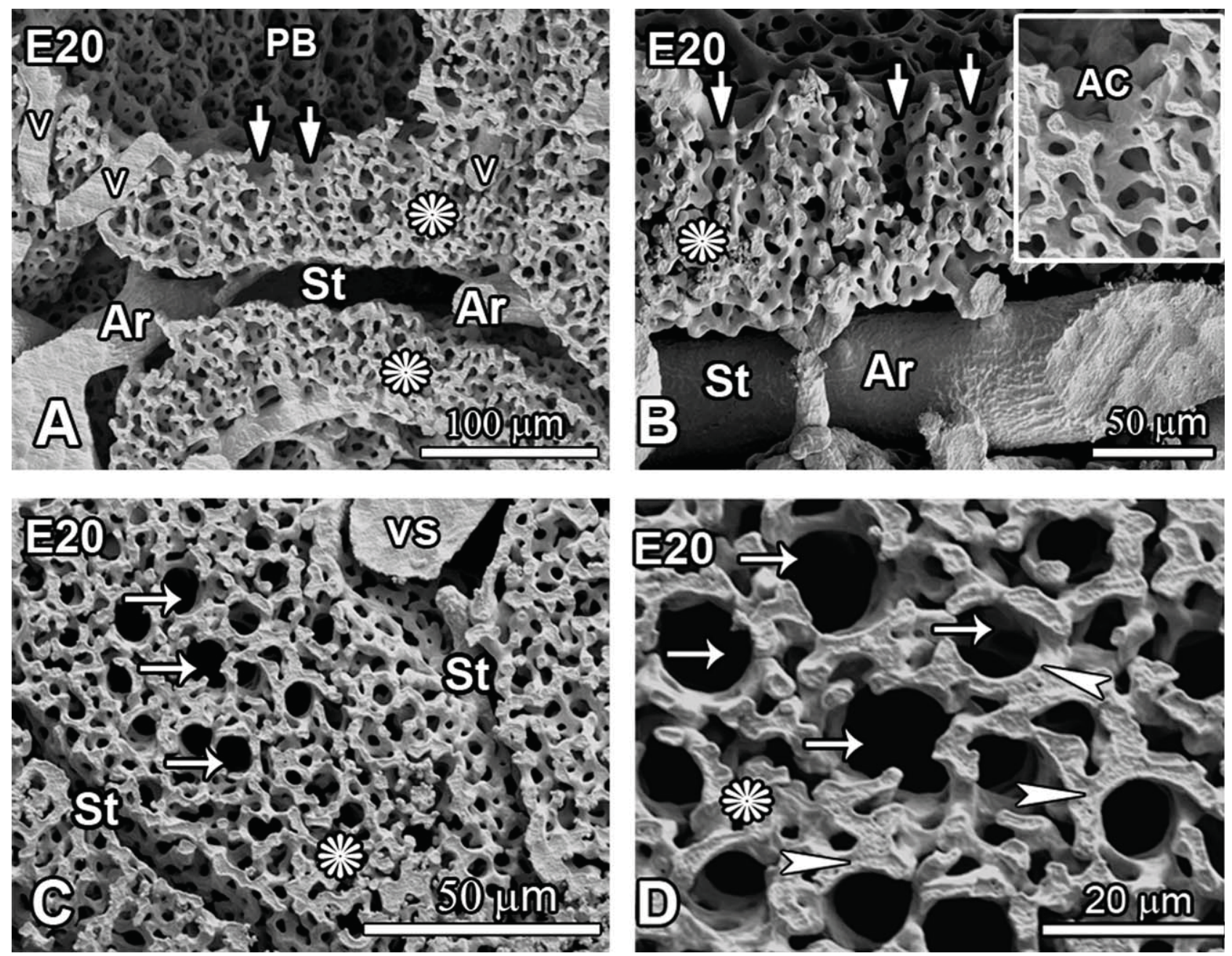

Fig. 5. SEM micrographs of intravascular lung casts showing the 3D disposition of blood capillaries around the lumina of air capillaries at E20. A and B: Casts sectioned parallel to the long axes of the air capillaries. The ACs (arrows) spread from the parabronchial lumen (PB) and pierce the parabronchial capillary mantle toward the septum (St). The interparabronchial septum has the large arteries (Ar). The parabronchial mantle (asterisks) is traversed by intraparabronchial veins (V). Inset in B shows the net-like blood capillary meshwork surrounding an individual air capillary (AC). C: Casts sectioned at the periphery of the parabronchial mantle to show the interparabronchial septum (St) with a large interparabronchial vessel (Vs) and air capillaries (arrows) that extend to the periphery of the blood capillary meshwork (asterisk). D: A close-up of the transected BCs at the periphery of the parabronchial mantle shows that BCs tend to form complete rings (arrowheads) that surround the ACs, accomplishing a cross-current system. In between the ACs, the BCs form a dense network (asterisk).

generally run perpendicular to the long axes of the parabronchi, there is no apparent functional significance in this arrangement and other vessel generations run in varied directions (Makanya and Djonov, 2009). Nevertheless, some authors have used this arrangement to describe the system as being cross-current (Maina, 2006), in contrast to the original model of Scheid and Piiper (1972).

The original cross-current model described by Scheid and Piiper (1972) was based on the relationship between bulk convective gas flow in the parabronchial lumen and the venous blood flowing centripetally in the parabronchial capillaries. This arrangement was conceptualized to be in such a way that the parabronchial gas flow was perpendicular to the parabronchial capillary blood flow. Measurements of gas partial pressures in the air exiting the lungs as well as the postpulmonary oxygenated blood showed that it was possible to have higher oxygen concentrations in the blood exiting the lungs than in the exhaled air. For this reason, and the perceived relationship between the parabronchial air and parabronchial capillary blood, the system was dubbed cross-current exchange (Fedde, 1998; Scheid and Piiper, 1972).

The gas exchange system in the avian lung has been postulated to be cross-current, proved physiologically and envisages the convective airflow within the parabronchus being perpendicular to the centripetal flow of the blood in the parabronchial capillaries (Farmer, 2006; Scheid and Piiper, 1972). The general direction taken by the venous blood is actually centripetal, where it flows from the interparabronchial arteries via the intricate parabronchial capillary meshwork to the venous system at the parabronchial lumen. The direction of flow, however, changes at the AC level to become orthogonal to that of the air in the ACs. These 

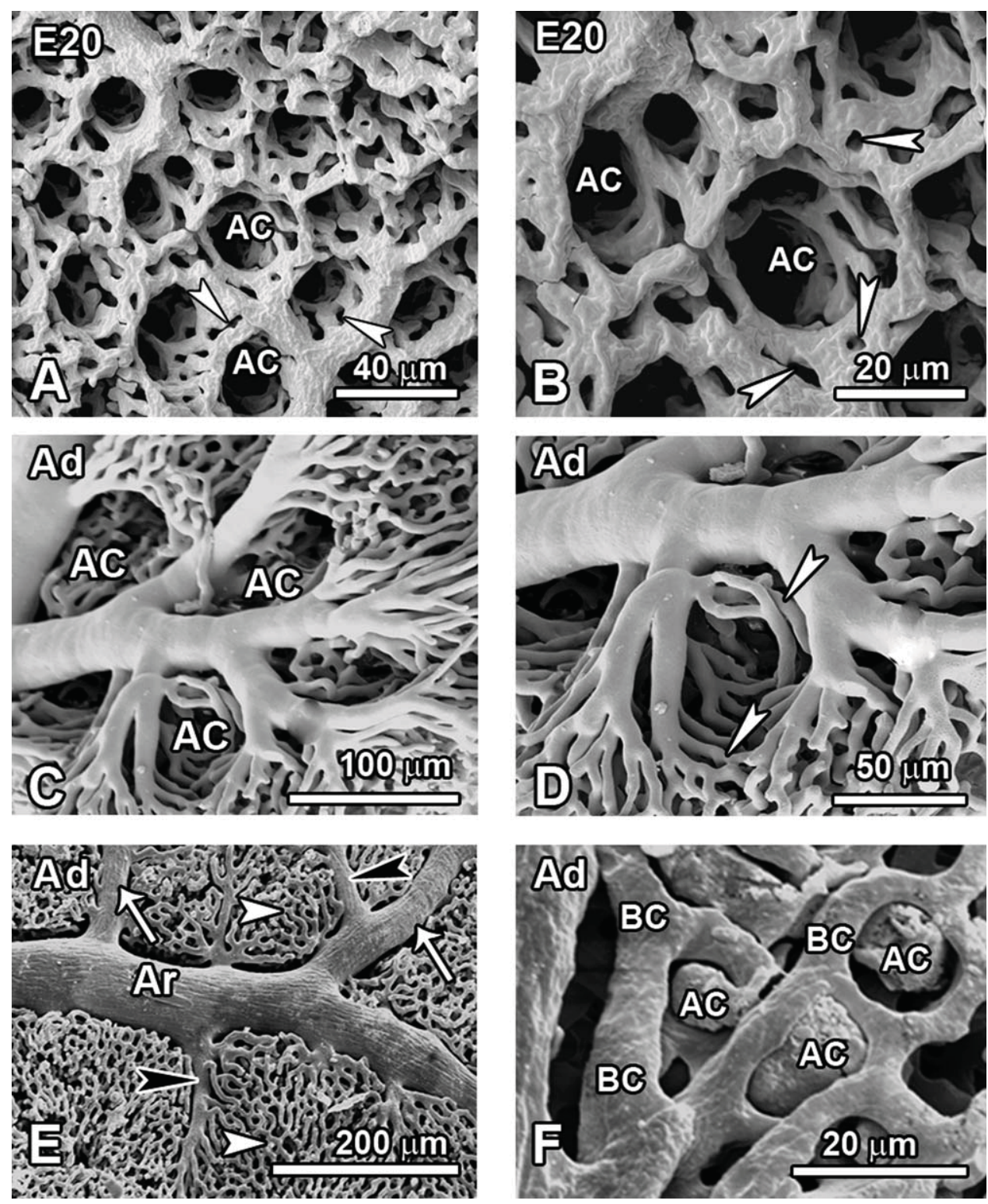

Fig. 6. SEM micrographs of intravascular lung casts comparing the spatial arrangement of the blood capillaries around the lumina of air capillaries in the prehatch chick embryo (E20; A and B) and the adult (C and D). An intravascular cast and a double cast in the adult are presented in E and F, respectively. A and B: Viewed from the parabronchial lumen, the blood capillaries at E20 are composed of net-like baskets surrounding the air capillaries (AC). These capillaries are of irregular girth and the casts are characterized by numerous holes (open arrowheads), which represent tissue pillars characteristic of intussusceptive angiogenesis. $\mathbf{C}$ and $\mathbf{D}$ : Luminal view of vascular casts in the adult bird reveals a totally different picture with the BCs forming smooth tubular structures (open arrowheads in $\mathrm{D}$ ) running perpendicular to the long axes of the ACs and surrounding the ACs in such a manner that a cross-current system is established. Notably these capillaries are virtually devoid of tissue pillars, as indicated by absence of holes on the vascular casts. $\mathbf{E}$ and $\mathbf{F}$ : Overview of the external aspect of the parabronchial vascular casts. Note the close-knit capillary network (open arrowheads in E) that covers the gas exchange tissue. The large arteries (Ar) run in the interparapronchial septum and give rise to parabronchial arteries (open arrows) and parabronchial arterioles (closed arrowheads). Notice the exchange blood capillaries surrounding the tips of ACs (BC in F). Such BCs form arcs around the ACs, highlighting the tendency to maintain a cross-current system even at the peripheral parts of the parabronchial mantle.

exchange BCs then gather to form the venous system, which starts with the infundibular and septal veins. The latter category of veins converge to form atrial veins that drain into the intraparabronchial veins, the only veins this far reported to traverse the parabronchial mantle (Makanya and Djonov, 2009). The angioarchitecture of the parabronchial vessels has recently been documented in generous details
(Makanya and Djonov, 2009), and embryonic development of the avian pulmonary vasculature was reported previously (Anderson-Berry et al., 2005; Makanya et al., 2007).

A few reports have suggested that the exchange at the AC-BC interface is cross-current (Herenda and Franco, 1999; Makanya and Djonov, 2009; Nasu, 2005; West et al., 1977), but concrete evidence has not been 
documented. Previously, the assumption was that the interaction between the ACs and BCs within the parabronchial mantle was counter-current (Duncker, 1971; Dyce et al., 2002; Maina, 2006). We demonstrated earlier that the blood flows from the interparabronchial arteries from the periphery and passes through the bulk of the parabronchial capillaries to reach the veins at the interior aspect of the parabronchus and then exits via the intraparabronchial veins to join the interparabronchial veins (Makanya and Djonov, 2009). Here, we show that the bulk of these are oriented in many directions with respect to the long axis of the parabronchus but are essentially perpendicular to the long axes of the ACs. Surprisingly, recent investigations continue to dispute the existence of a cross-current system at the AC level, without any tangible evidence to the contrary (Maina and Woodward, 2009).

The mechanisms through which gas traverses into and out of the ACs are at the moment unclear, but the prominent atrial muscles in the interatrial septa probably play a role in the pressure changes within the air conduits. Indeed Duncker (1978) notes that the atrial muscles regulate air flow by varying parabronchial diameter, and it is plausible that in the same way they may regulate flow in and out of the atria (Dyce et al., 2002).

Precise understanding of the functioning of the avian lung has been stymied by incomplete knowledge of the functional structure and the confusion in the literature further confounds the problem. In the domestic fowl, the ACs are reported to be narrow tubes that branch and anastomose freely with each other (Dyce et al., 2002), while elsewhere they are reported to be rotund structures that anastomose profusely through narrow passages (Maina, 2006). Other reports indicate that the ACs of the domestic fowl, one of the extensively studied avian species, are highly anastomosing non blind-ending tubules (Fujii et al., 1981; Maina and King, 1982). Because of the widespread confusion regarding the structure of the avian lung, the current data have been presented with abundant pictorial evidence, in an attempt to stave off any further confusion. Previously, we have indicated that to unambiguously elucidate the complex structure of the avian lung, more than one visualization techniques are necessary and that studying the structures during their formative stages better illuminates the final topography (Makanya and Djonov, 2008).

The cross-current design of the avian lung as initially conceptualized relates to convective gas flow in the parabronchial lumen and the blood flow in the exchange capillaries. The design was visualized using a physiological cross-current model (Fedde, 1998; Powell and Scheid, 1989) with little empirical data. The entrance of the mixed venous blood from the pulmonary arteries along the periphery of the parabronchial mantle with BCs in intimate contact with ACs, and collection of the arterialized blood in venules located immediately beneath the epithelium of the lumen of the parabronchus (Abdalla and King, 1975; King and McLelland, 1984) was considered to be orthogonal to the convective flow of gas. Blood entering a parabronchus at its origin from a laterodorsal [formerly mediodorsal, see (Makanya and Djonov, 2008)] secondary bronchi equilibrates with the high $\mathrm{PO}_{2}$ and low $\mathrm{PCO}_{2}$ in the gas while blood near the end of the parabronchus equilibrates with a gas that contains a much lower $\mathrm{PO}_{2}$ and higher $\mathrm{PCO}_{2}$. Thus, it is possible for the blood leaving the exchanger to have a higher $\mathrm{PO}_{2}$ than that in the gas leaving the exchanger. This led to the model being designated cross-current (Fedde, 1998; Piiper, 1982; Powell and Scheid, 1989; Scheid and Piiper, 1972) and signifies a gas exchanging system with an inherently high efficacy. The combination of the cross-current arrangement at the AC-BC interface and the arrangement referred to as multicapillary serial arterialization (Maina, 2006) combine to give the avian lung a high degree of efficiency.

In the current study, we have used no less than seven visualization techniques (see Table 1) to resolve the controversy regarding the $3 \mathrm{D}$ structure of $\mathrm{ACs}$ and $\mathrm{BCs}$, and have shown the presence of a predominantly cross-current arrangement at the gas exchange interface. BCs tend to circumferentially surround the infundibulae and the ACs, a situation demonstrated previously (Makanya and Djonov, 2009; Nasu, 2005; West et al., 1977), changing direction toward the atrial opening where they converge to form the small venules that join the hierarchy of veins draining the parabronchus (Makanya and Djonov, 2009). Although some investigators expressed doubt over casting methods (Maina and Woodward, 2009), it is noteworthy that techniques employing highly refined resins such as mercox are able to reproduce fine structures of both ACs and BCs. The reports by West et al. (1977), Nasu (2005), and Makanya and Djonov (2009) clearly represent the BCs both in terms of size and spatial disposition and these studies point to a cross-current arrangement at the exchange interface. Indeed intravascular casting was the method used to discover the existence of intussusceptive angiogenesis, a novel mechanism of vascular growth and remodeling that proceeds through formation of tiny $(\approx 2 \mu \mathrm{m})$ intraluminal tissue pillars in capillaries (Caduff et al., 1986; Makanya et al., 2009), and which is extremely important in vascular patterning and establishment of the ultimate organ-specific angioarchitecture (Makanya et al., 2009). Although each visualization technique has its own limitations, a combination of several such techniques helps to resolve the problem (Makanya and Djonov, 2008).

Besides the efficiency in gas exchange, the 3D arrangement between the ACs and BCs is important in lending mechanical strength to each other (Watson et al., 2008; West, 2009; West et al., 2006), and allows an extremely thin albeit strong blood-gas barrier (Maina and West, 2005; West, 2009). However, whether the cross-current arrangement has any implications on the mechanical strength of these gas-exchanging units remains to be investigated. The current findings nevertheless, provide empirical data for further physiological investigations.

\section{ACKNOWLEDGMENTS}

The authors thank Brigitte Scolari, Regula Buergy, Krystyna Sala, Bettina de Breuyn, Barbara Krieger, Irene Osoro, Christoph Lehmann, George Kariuki, Peter Kiguru, Francis Okumu, and John Kiai for their excellent technical assistance. 


\section{REFERENCES}

Abdalla MA, King AS. 1975. The functional anatomy of the pulmonary circulation of the domestic fowl. Respir Physiol 23:267290.

Anderson-Berry A, O'Brien EA, Bleyl SB, Lawson A, Gundersen N, Ryssman D, Sweeley J, Dahl MJ, Drake CJ, Schoenwolf GC, Albertine KH. 2005. Vasculogenesis drives pulmonary vascular growth in the developing chick embryo. Dev Dyn 233:145-153.

Caduff JH, Fischer LC, Burri PH. 1986. Scanning electron microscope study of the developing microvasculature in the postnatal rat lung. Anat Rec 216:154-164.

Duncker HR. 1971. The lung-air sac system of birds. A contribution to the functional anatomy of the respiratory apparatus. Ergeb Anat Entwicklungsgesch 45:7-171.

Duncker HR. 1978. General morphological principles of amniotic lungs. In: Piiper J, editor. Respiratory function in birds, adult and embryonic. Heidelberg: Springer-Verlag. pp.1-15.

Dyce KM, Wolfgang S, Wensig CJG. 2002. Textbook of veterinary anatomy. Philadelphia: WB Saunders.

Farmer CG. 2006. On the origin of avian air sacs. Respir Physiol Neurobiol 154:89-106.

Fedde MR. 1998. Relationship of structure and function of the avian respiratory system to disease susceptibility. Poult Sci 77:11301138

Fujii S, Tamura T, Okamoto T. 1981. Microarchitecture of air capillaries and blood capillaries in the respiratory area of the hen's lung examined by scanning electron microscopy. Nippon Juigaku Zassh 43:83-88.

Herenda DC, Franco DA. 1999. Poultry diseases and meat hygiene: A color atlas. Iowa: Iowa State University Press.

King AS, McLelland J. 1984. Birds: Their structure and function. London: Ballie're Tindall.

Klika E, Scheuermann DW, De Groodt-Lasseel MH, Bazantova I, Switka A. 1997. Anchoring and support system of pulmonary gas-exchange tissue in four bird species. Acta Anat (Basel) 159:3041.

Klika E, Scheuermann DW, De Groodt-Lasseel MH, Bazantova I, Switka A. 1998. An SEM and TEM study of the transition of the bronchus to the parabronchus in quail (Coturnix coturnix). Ann Anat 180:289-297.

Locy WA, Larsell O. 1916. The embryology of the bird's lung based on observations of the domestic fowl: Part II. Am J Anat 20:1-44.

Maina JN. 2003. A systematic study of the development of the airway (bronchial) system of the avian lung from days 3 to 26 of embryogenesis: A transmission electron microscopic study on the domestic fowl, Gallus gallus variant domesticus. Tissue Cell 35:375-391.

Maina JN. 2006. Development, structure, and function of a novel respiratory organ, the lung-air sac system of birds: To go where no other vertebrate has gone. Biol Rev Camb Philos Soc 81:545-579.

Maina JN. 2008. Functional morphology of the avian respiratory system, the lung-air sac system: Efficiency built on complexity Ostrich: J Afr Ornithol 79:117-132.

Maina JN, King AS. 1982. Morphometrics of the avian lung. II. The wild mallard (Anas platyrhynchos) and graylag goose (Anser anser). Respir Physiol 50:299-310.
Maina JN, West JB. 2005. Thin and strong! The bioengineering dilemma in the structural and functional design of the blood-gas barrier. Physiol Rev 85:811-844.

Maina JN, Woodward JD. 2009. Three-dimensional serial section computer reconstruction of the arrangement of the structural components of the parabronchus of the Ostrich, Struthio camelus lung Anat Rec (Hoboken) 292:1685-1698.

Makanya AN, Djonov V. 2008. Development and spatial organization of the air conduits in the lung of the domestic fowl, Gallus gallus variant domesticus. Microsc Res Tech 71:689-702.

Makanya AN, Djonov V. 2009. Parabronchial angioarchitecture in developing and adult chickens. J Appl Physiol 106:1959-1969.

Makanya AN, Hlushchuk R, Duncker HR, Draeger A, Djonov V. 2006. Epithelial transformations in the establishment of the blood-gas barrier in the developing chick embryo lung. Dev Dyn 235:68-81.

Makanya AN, Hlushchuk R, Baum O, Velinov N, Ochs M, Djonov V. 2007. Microvascular endowment in the developing chicken embryo lung. Am J Physiol Lung Cell Mol Physiol 292:L1136-L1146.

Makanya AN, Hlushchuk R, Djonov VG. 2009. Intussusceptive angiogenesis and its role in vascular morphogenesis, patterning, and remodeling. Angiogenesis 12:113-123.

Nasu T. 2005. Scanning electron microscopic study on the microarchitecture of the vascular system in the pigeon lung. J Vet Med Sci 67:1071-1074.

Piiper J. 1982. Respiratory gas exchange at lungs, gills and tissues: Mechanisms and adjustments. J Exp Biol 100:5-22.

Powell FL, Scheid P. 1989. Physiology of gas exchange in the avian respiratory system. In: King AS, McLelland J, editors. Form and function in birds. London: Academic press. pp.393-437.

Scheid P, Piiper J. 1972. Cross-current gas exchange in avian lungs: Effects of reversed parabronchial air flow in ducks. Respir Physiol 16:304-312

Scheuermann DW, Klika E, De Groodt-Lasseel MH, Bazantova I, Switka A. 1997. An electron microscopic study of the parabronchial epithelium in the mature lung of four bird species. Anat Rec 249:213-225.

Scheuermann DW, Klika E, Groodt-Lasseel MH, Bazantova I, Switka A. 1998. The development and differentiation of the parabronchial unit in quail (Coturnix coturnix). Eur J Morphol 36:201-215.

Watson RR, Fu Z, West JB. 2008. Minimal distensibility of pulmonary capillaries in avian lungs compared with mammalian lungs. Respir Physiol Neurobiol 160:208-214.

West JB. 2009. Comparative physiology of the pulmonary blood-gas barrier: The unique avian solution. Am J Physiol Regul Integr Comp Physiol 297:R1625-R1634.

West JB, Watson RR, Fu Z. 2006. The honeycomb-like structure of the bird lung allows a uniquely thin blood-gas barrier. Respir Physiol Neurobiol 152:115-118

West NH, Bamford OS, Jones DR. 1977. A scanning electron microscope study of the microvasculature of the avian lung. Cell Tissue Res 176:553-564.

Woodward JD, Maina JN. 2005. A 3D digital reconstruction of the components of the gas exchange tissue of the lung of the muscovy duck, Cairina moschata. J Anat 206:477-492.

Woodward JD, Maina JN. 2008. Study of the structure of the air and blood capillaries of the gas exchange tissue of the avian lung by serial section three-dimensional reconstruction. J Microsc 230:84-93. 